\title{
Sensitizing multi drug resistant Staphylococcus aureus isolated from surgical site infections to antimicrobials by efflux pump inhibitors
}

\author{
Baiomy Amr A, Shaker Ghada H, Abbas Hisham A
}

Zagazig University, Faculty of pharmacy, Department of microbiology and Immunology.

\begin{abstract}
Background: Staphylococcus aureus is a common hospital acquired infections pathogen. Multidrug-resistant Methicillin-resistant Staphylococcus aureus represents a major problem in Egyptian hospitals. The over-expression of efflux pumps is a main cause of multidrug resistance. The discovery of efflux pump inhibitors may help fight multidrug resistance by sensitizing bacteria to antibiotics. This study aimed to investigate the role of efflux pumps in multidrug resistance.

Methods: Twenty multidrug resistant $S$. aureus isolates were selected. Efflux pumps were screened by ethidium bromide agar cartwheel method and polymerase chain reaction. The efflux pump inhibition by seven agents was tested by ethidium bromide agar cartwheel method and the effect on sensitivity to selected antimicrobials was investigated by broth microdilution method.

Results: Seventy percent of isolates showed strong efflux activity, while $30 \%$ showed intermediate activity. The efflux genes $m d e A$, nor $B$, nor $C$, nor $A$ and $\operatorname{sep} A$ were found to play the major role in efflux, while genes mep $A, \operatorname{smr}$ and $q a c A / B$ had a minor role. Verapamil and metformin showed significant efflux inhibition and increased the sensitivity to tested antimicrobials, while vildagliptin, atorvastatin, domperidone, mebeverine and nifuroxazide showed no effect.

Conclusion: Efflux pumps are involved in multidrug resistance in Staphylococcus aureus. Efflux pump inhibitors could increase the sensitivity to antimicrobials.

Keywords: Staphylococcus aureus; multidrug resistance; efflux pump inhibitors.

DOI: https://dx.doi.org/10.4314/ahs.v20i4.16

Cite as: Baiomy AA, Shaker GH, Abbas HA. Sensitizing multi drug resistant Staphylococcus aureus isolated from surgical site infections to antimicrobials by efflux pump inbibitors. Afri Health Sci. 2020;20(4):1632-45. https:// dx.doi.org/10.4314/ abs.v20i4.16
\end{abstract}

\section{Introduction}

Surgical site infections (SSIs) are those infections that affect either the skin or the underlying soft tissues at the surgical site with a primarily closed incision that occurs within 30 days post-surgery ${ }^{1}$.

Resistance of Staphylococcus aureus ( $S$. aureus) to antimicrobial agents can take place by intrinsic or acquired resistance ${ }^{2}$. The emergence of multi-drug resistance may indicate the presence of efflux pumps ${ }^{3}$. Active efflux contributes to the antimicrobial resistance of many bacteria ${ }^{4}$. Bacterial efflux systems are members of larger classes of transporters responsible for the uptake of essential nutrients and ions, removal of end metabolites, harmful substances and communication between cells and the environment ${ }^{5}$.

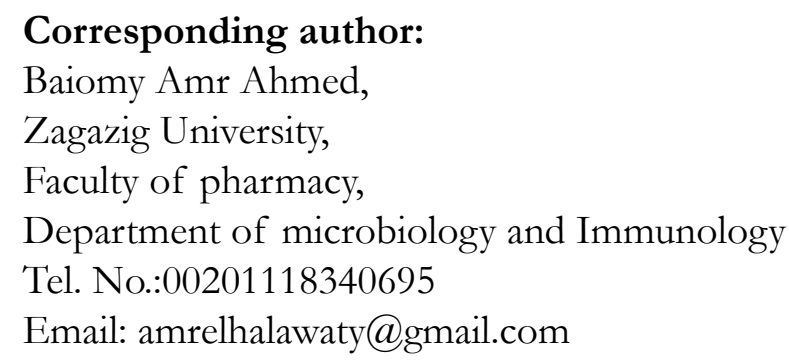

In Gram-positive bacteria, efflux pumps fall into four unrelated families; small multi-drug resistance (SMR), major facilitator superfamily (MFS), adenosine triphosphate (ATP) binding cassette (ABC) and multi-drug and toxic extrusion (MATE)

To reverse efflux pump-mediated antimicrobial resistance, compounds that can inhibit efflux pumps activities; efflux pump inhibitors (EPIs) are necessary in order to treat infections caused by multidrug resistant (MDR) bacteria $^{7}$. These compounds can be used in combination with the relevant antibiotics to achieve synergistic action. Previous studies showed that many synthetic and natural compounds can be used for this purpose. Among natural compounds, quercetin, pyrogallol and tannic acid could inhibit NorA efflux pump in $S$. aureus and the pyrogallol could potentiate gentamicin and norfloxacin against $S$. aureus ${ }^{8-10}$. Moreover, some thiazolidinedione and thiazole derivatives were found to augment noffloxacin by interference with the activity of NorA efflux pump ${ }^{11}$.

The objective of this study was the detection of efflux pump as a mechanism of resistance to antimicrobials in some (MDR) $S$. aureus isolates in addition to screening 
for some new agents that could act as EPIs and potentiate the effect of antimicrobial agents.

\section{Materials and methods \\ Bacterial strains}

Twenty MDR $S$. aureus isolates were selected from our previous work $^{12}$. They were obtained from patients with SSIs admitted to Surgery Department in Zagazig University Hospitals, Zagazig, Egypt.

\section{Media and chemicals}

Mueller Hinton agar and broth, Tryptone soya agar, Nutrient agar and broth and agar were obtained from Oxoid, Hampshire, England in dehydrated form.

Domperidone, atorvastatin, nifuroxazide, sulfamethoxazole-trimethoprim, ampicillin-sulbactam and mebeverine were obtained from Egyptian Pharmaceutical Industries Company (EPICO), Egypt, while vildagliptin and metformin were obtained from National Pharmaceutical Company (NAPCO), Egypt. Imipenem as Tienam ${ }^{\circledR}$ powder for IV infusion was the marketed pharmaceutical product manufactured by Merck \& Co., Inc, USA. Linezolid, levofloxacin, amikacin and doxycycline were obtained from PHARCO Company, Egypt. Clindamycin was kindly granted from PFIZER Company. PCR master mix, Agarose DNA grade, DNA marker (100 bp), Tris-acetate-EDTA (TAE) buffer (50X), verapamil, vancomycin and the PCR primers ${ }^{13}$ (table 1$)$, provided by Integrated DNA Technologies were purchased from Sigma Aldrich, St. Louis, Mo, USA. DNase free water used for PCR reaction mixture was the product of Thermo Fisher Scientific, USA.

\section{Methods}

Phenotypic assessment for efflux pumps in MDR $S$. aurens isolates by ethidium bromide cartwheel method (EtBrCw) method

In order to investigate the presence of efflux pumps, the ethidium bromide cartwheel method was used. Ethidium bromide $(\mathrm{EtBr})$ is an intercalating fluorescent dye that can traverse the bacterial cell wall. After entry, the dye can accumulate to a point that induces fluorescence on excitation by ultraviolet (UV) light. MDR Efflux pumps can extrude this substrate to the medium. As the efflux capacity of the cells increases, the concentration of the dye needed to produce fluorescence and the emitted fluorescence can be used to assess the efflux capacity of the isolates ${ }^{14}$.

Trypticase Soya Agar (TSA) plates were freshly prepared on the same day of the experiment and they contained different ethidium bromide concentrations ( 0.0 to $3 \mathrm{mg} / \mathrm{L}$ ). The plates were kept protected from light.
Each isolate was grown overnight prepared in Mueller-Hinton broth and the turbidities of the resultant suspensions were adjusted to $0.5 \mathrm{McF}$ arland standard. To form a cartwheel pattern, TSA plates were divided into eight to ten sectors radially. The adjusted bacterial inocula were surface inoculated on the EtBr-TSA plates and the TSA plates were incubated at $37^{\circ} \mathrm{C}$ for 16 hrs. TSA plates were examined under UV transilluminator and the least concentration of $\mathrm{EtBr}$ that induced fluorescence of the bacterial mass was detected and the plates were photographed. Isolates that produced fluorescence at concentrations more than $2 \mu \mathrm{g} / \mathrm{ml} \mathrm{EtBr}$ were considered to have positive efflux activity, while the fluorescence at concentrations less than or equal to $1 \mu \mathrm{g} / \mathrm{ml} \mathrm{EtBr}$ indicated negative efflux activity. On the other hand, the presence of fluorescence at concentrations equal to $2 \mu \mathrm{g} / \mathrm{ml} \mathrm{EtBr}$ indicated intermediate efflux activity ${ }^{15,16}$.

Genotypic screening for efflux pump genes by PCR

All twenty $S$. aureus isolates were tested for the presence of six chromosomal encoded genes (nor A, norB, norC, sepA, mepA, mdeA) and two plasmid encoded genes (qacA/B and smr) encoding efflux pumps in S. aureus by polymerase chain reaction (PCR) amplification technique ${ }^{14,17}$.

The crude cell lysate was prepared according to Nair and Venkitanarayanan ${ }^{18}$. Two or three colonies of overnight cultures of each isolate on nutrient agar were suspended in $50 \mu \mathrm{L}$ PCR-quality water and then heated at $95^{\circ} \mathrm{C}$ for 10 minutes in PCR thermocycler. After quick centrifugation of the bacterial suspensions at $13000 \mathrm{rpm}$ for 30 seconds, supernatants containing the genomic DNA were collected and stored at $-20^{\circ} \mathrm{C}$ to be used in the PCR. Two $\mu$ l of the prepared extract of each isolate was used as DNA template for PCR amplification. The amplification mixture was prepared in a final volume of $25 \mu \mathrm{L}$ and consists of PCR Mix MyTaq ${ }^{\text {TM }}$ red Master Mix $(2 \mathrm{X})(12.5 \mu \mathrm{L}$, forward primer $(1.5 \mu \mathrm{L})$, reverse primer $(1.5 \mu \mathrm{L})$, template DNA $(2 \mu \mathrm{L})$, nuclease-free water $(7.5 \mu \mathrm{L})$. The DNA amplification conditions were as follows:

DNA was denatured at $94^{\circ} \mathrm{C}$ for $4 \mathrm{~min}$, followed by 35 cycles of denaturation at $94^{\circ} \mathrm{C}$ for $30 \mathrm{~s}$. The annealing temperatures were $60^{\circ} \mathrm{C}$ for nor $\mathrm{A}, 62^{\circ} \mathrm{C}$ for norB and norC and $61^{\circ} \mathrm{C}$ for sepA, mepA and mdeA for 45-55 s. The step of extension was carried out at $72{ }^{\circ} \mathrm{C}$ for 55 s-1 min, and was followed by a step of final extension at $72{ }^{\circ} \mathrm{C}$ for $5 \mathrm{~min}$. furthermore, the PCR reactions for genes qacA/B and smr were performed under the following conditions: denaturation of DNA at $95^{\circ} \mathrm{C}$ for $1 \mathrm{~min}$, followed by 30 cycles of denaturation at $95^{\circ} \mathrm{C}$, 
each for $1 \mathrm{~min}$, annealing at $58^{\circ} \mathrm{C}$ for qacA/B and smr for $45 \mathrm{~s}$ and extension at $72{ }^{\circ} \mathrm{C}$ for $1 \mathrm{~min}$, and finally a step of final extension at $72{ }^{\circ} \mathrm{C}$ for $5 \mathrm{~min}$. The amplification products were electrophoresed in agarose gel $(1 \%)$ for $1 \mathrm{~h}$ at $100 \mathrm{~V}$, stained with $\mathrm{EtBr}$ of concentration $0.5 \mathrm{mg} / \mathrm{ml}^{19}$, and photographed under UV light.

Determination of minimum inhibitory concentration (MIC) of different antibiotics biocides and potential efflux pump inhibitors against $S$. aureus isolates

To determine the minimum inhibitory concentration, the broth microdilution method was used following the CLSI guidelines and Vicki al. ${ }^{20,21}$. A suspension of four morphologically similar colonies for each isolate was made in sterile saline in order to have a turbidity matching that of $0.5 \mathrm{McF}$ arland Standard. The resulting suspensions were diluted 1:100 in Mueller Hinton broth. Different two fold serially diluted antimicrobial solutions or drugs (vildagliptin, metformin, atorvastatin, domperidone, mebeverine and nifuroxazide) that were screened for their potential efflux pump inhibitory activity were made in the wells of 96-well microtiter plate so that each well contains $50 \mu$ l of the antibiotic dilution. To the antibiotic-containing wells, aliquots of $50 \mu \mathrm{l}$ of suspension were transferred. The plates were incubated at $35-37^{\circ} \mathrm{C}$ for $18-24 \mathrm{hrs}$, and the MIC was calculated by observing the lowest concentration of the antimicrobial that inhibited visible growth of the microorganism. The antibiotics used were vancomycin, linezolid, ampicillin-sulbactam, levofloxacin, doxycy- cline, amikacin, sulfamethoxazole-trimethoprim, clindamycin and imipenem. The biocides used included povidone iodine, glutaraldehyde, cetrimide, chlorhexidine and crystal violet.

Screening for the effect of the some agents on efflux pumps activity by $\mathrm{EtBrCw}$ method

The cartwheel assay was used in the presence of $1 / 4$ MIC of selected drugs and the fluorescence was recorded and compared to that produced in control plates without the tested drugs. Verapamil was used as well reported control EPI, where other drugs (vildagliptin, metformin, atorvastatin, domperidone, mebeverine and nifuroxazide) were used as potential efflux pump inhibitors.

Effect of potential efflux pump inhibitors (EPIs) on the resistance of $S$. aureus isolates to different antimicrobial agents

In order to confirm the effect of the potential EPIs, the MICs of tested antibiotics and biocides were determined by broth microdilution method in the presence of $1 / 4$ MIC (sub-MIC) of the tested agents against $S$. aureus isolates.

\section{Results}

Phenotypic assessment for efflux pumps in MDR $S$. aureus isolates by $\mathrm{EtBrCw}$ method

Fourteen resistant isolates $(70 \%)$ were EtBrCW-positive and six isolates $(30 \%)$ were EtBrCW-intermediate. The EtBrCW method was used to screen for efflux pump activity (table 2 and Fig. 1). 


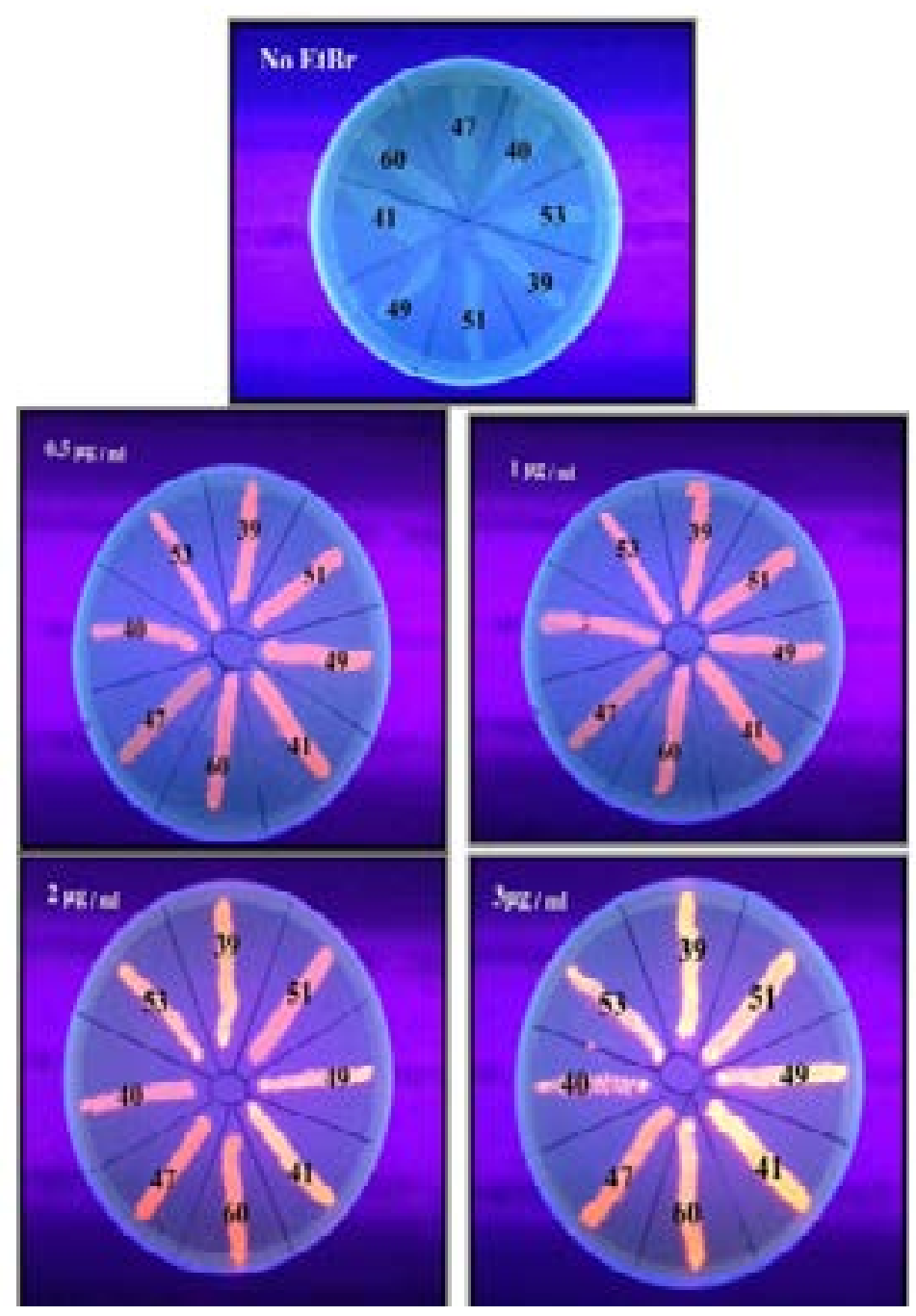

Figure 1. Qualitative assessment of efflux pumps activity by EtBr agar cartwheel method. Isolates that produced fluorescence at concentrations more than $2 \mu \mathrm{g} / \mathrm{ml} \mathrm{EtBr}$ were considered to have positive efflux activity, while the fluorescence at concentrations less than or equal to $1 \mu \mathrm{g} / \mathrm{ml}$ EtBr indicated negative efflux activity. On the other hand, the presence of fluorescence at concentrations equal to $2 \mu \mathrm{g} / \mathrm{ml} \mathrm{EtBr}$ indicated intermediate efflux activity. Isolates No. 39, 41, 49 and 53 showed emission of fluorescence at EtBr concentration $2 \mu \mathrm{g} / \mathrm{ml}$ (CW intermediate), while isolates 51,60 , 47 and 40 showed no fluorescence at that concentration but showed fluorescence at $3 \mu \mathrm{g} / \mathrm{ml}$ except isolate No. 47 which showed no fluorescence at that concentration (CW positive). 
Table 1. Primers used in PCR amplifications

\begin{tabular}{llll}
\hline Gene & Primer & Primer sequence (5-3) & Product size (bp) \\
\hline norA & $\mathrm{F}$ & TTCACCAAGCCATCAAAAAG & 620 \\
& $\mathrm{R}$ & CTTGCCTTTCTCCAGCAATA & \\
\hline norB & $\mathrm{F}$ & AGCGCGTTGTCTATCTTTCC & 213 \\
& $\mathrm{R}$ & GCAGGTGGTCTTGCTGATAA & \\
\hline norC & $\mathrm{F}$ & AATGGGTTCTAAGCGACCAA & 216 \\
& $\mathrm{R}$ & ATACCTGAAGCAACGCCAAC & \\
\hline sepA & $\mathrm{F}$ & GCAGTCGAGCATTTAATGGA & 103 \\
& $\mathrm{R}$ & ACGTTGTTGCAACTGTGTAAGA & \\
\hline mepA & $\mathrm{F}$ & ATGTTGCTGCTGCTCTGTTC & 718 \\
& $\mathrm{R}$ & TCAACTGTCAAACGATCACG & \\
\hline mdeA & $\mathrm{F}$ & AACGCGATACCAACCATTC & 677 \\
& $\mathrm{R}$ & TTAGCACCAGCTATTGGACCT & \\
\hline qacA/B & $\mathrm{F}$ & GCTGCATTTATGACAATGTTTG & 628 \\
& $\mathrm{R}$ & AATCCCACCTACTAAAGCAG & \\
\hline Smr & $\mathrm{F}$ & ATAAGTACTGAAGTTATTGGAAGT & 285 \\
& $\mathrm{R}$ & TTCCGAAAATGTTTAACGAAACTA & \\
& &
\end{tabular}

bp: base pair, F: forward primer, R: reverse primer

Table 2. Efflux pump activity of tested bacterial isolates by EtBr agar cartwheel method

\begin{tabular}{|c|c|c|c|c|c|}
\hline \multicolumn{6}{|c|}{$\operatorname{EtBr}(\mu \mathrm{g} / \mathrm{ml})$ and degree of fluorescence produced } \\
\hline Isolate No. & $0.5(\mu \mathrm{g} / \mathrm{ml})$ & $1(\mu \mathrm{g} / \mathrm{ml})$ & $2(\mu \mathrm{g} / \mathrm{ml})$ & $3(\mu \mathrm{g} / \mathrm{ml})$ & Efflux activity \\
\hline SA 27 & - & - & - & + & Positive \\
\hline SA 39 & - & - & + & + & Intermediate \\
\hline SA 40 & - & - & - & + & Positive \\
\hline SA 41 & - & - & + & + & Intermediate \\
\hline SA 44 & - & - & - & + & Positive \\
\hline SA 45 & - & - & - & + & Positive \\
\hline SA 47 & - & - & - & - & Positive \\
\hline SA 49 & - & - & + & + & Intermediate \\
\hline SA 50 & - & - & - & + & Positive \\
\hline SA 51 & - & - & - & + & Positive \\
\hline SA 53 & - & - & + & + & Intermediate \\
\hline SA 60 & - & - & - & + & Positive \\
\hline SA 77 & - & - & - & + & Positive \\
\hline SA 80 & - & - & - & + & Positive \\
\hline SA 81 & - & - & + & + & Intermediate \\
\hline SA 84 & - & - & - & + & Positive \\
\hline SA 85 & - & - & - & + & Positive \\
\hline SA 87 & - & - & - & - & Positive \\
\hline SA 89 & - & - & + & + & Intermediate \\
\hline SA 90 & - & - & - & + & Positive \\
\hline
\end{tabular}

SA: Staphylococcus aureus, $\because$ No fluorescence, +: Fluorescence. 
Genotypic screening for efflux pump genes by PCR For further identification of efflux pump as a resistance mechanism of the tested MDR $S$. aureus, the twenty MDR isolates were tested for the presence of efflux pump genes nor A, norB, norC, sepA, mepA, mdeA, qa$\mathrm{cA} / \mathrm{B}$ and smr genes in $\mathrm{S}$. aureus by PCR amplification technique.
In our study, 15 isolates $(75 \%)$ were positive for norA gene $(620 \mathrm{bp})$, while 18 isolates $(90 \%)$ and 16 isolates (80\%) showed norB (213 bp) and norC (216 bp) genes, respectively (Figs. 2-4). Moreover, sepA gene (103 bp) was found in 16 isolates $(80 \%)$. On the other hand, mepA gene (718 bp) was present in 8 isolates (40\%) (Figs. 5-6). Furthermore, mdeA gene (677 bp)was found in 18 isolates (90\%) (Fig. 7), while qacA/B gene (628 bp)was absent in all isolates and smr gene (285 bp) was found in only 2 isolates (10\%) (Figs. 8-9).

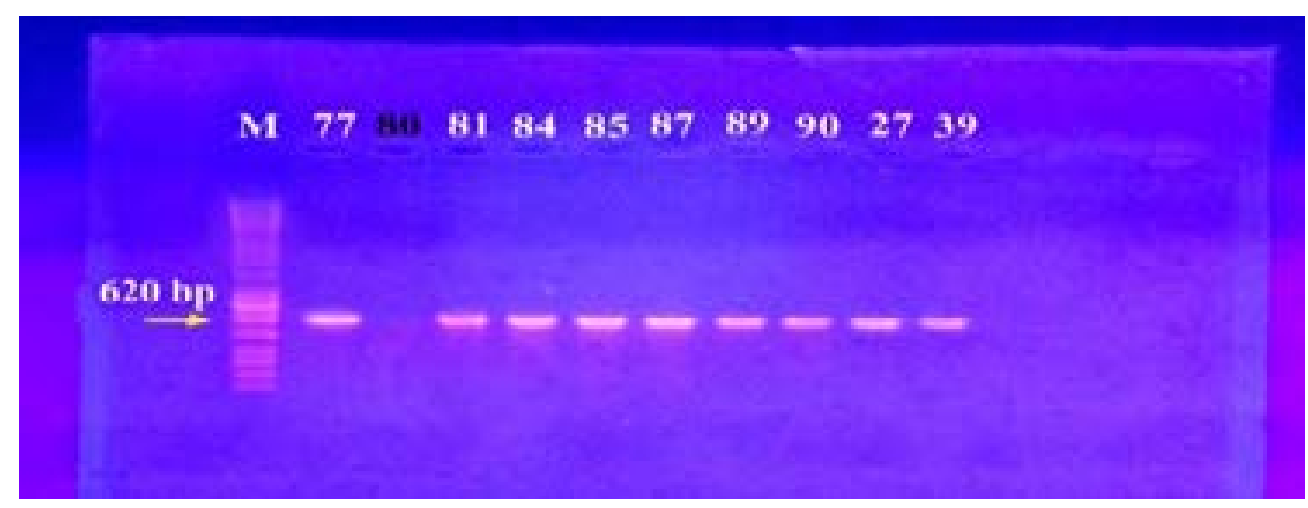

Figure 2. Electrophoresis of PCR products of norA gene. M; marker (100 bp).

Isolates $77,81,84,85,87,89,90,27$ and 39 showed a band at 620 bp that corresponds to norA gene.

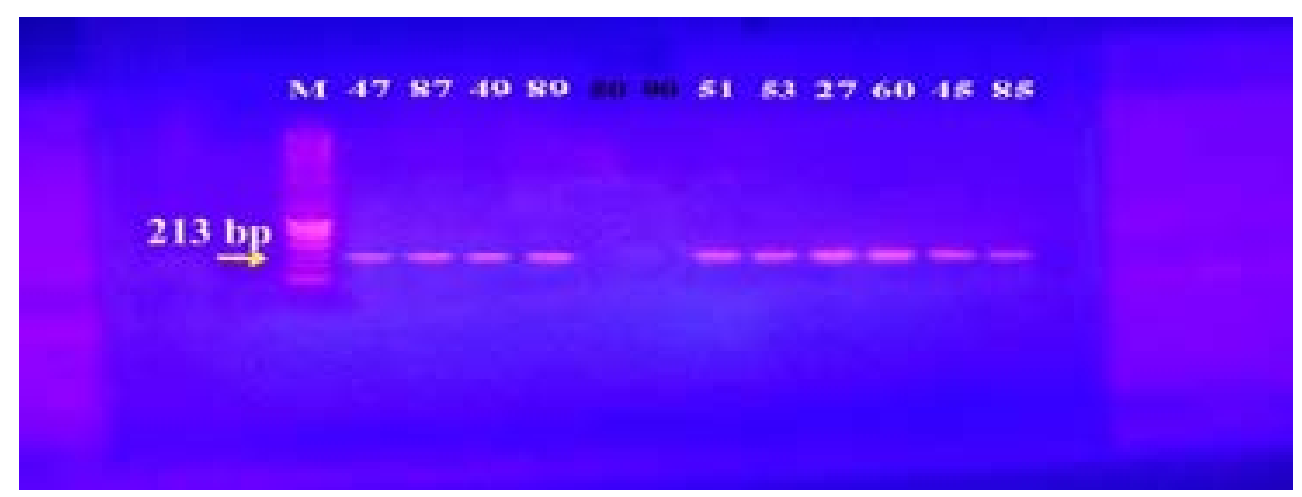

Figure 3. Electrophoresis of PCR products of norB gene. M; marker (100 bp), Isolates $47,87,49,89,51,53,27,60,45$ and 85 showed a band at $213 \mathrm{bp}$, that corresponds to $n o r B$ gene.

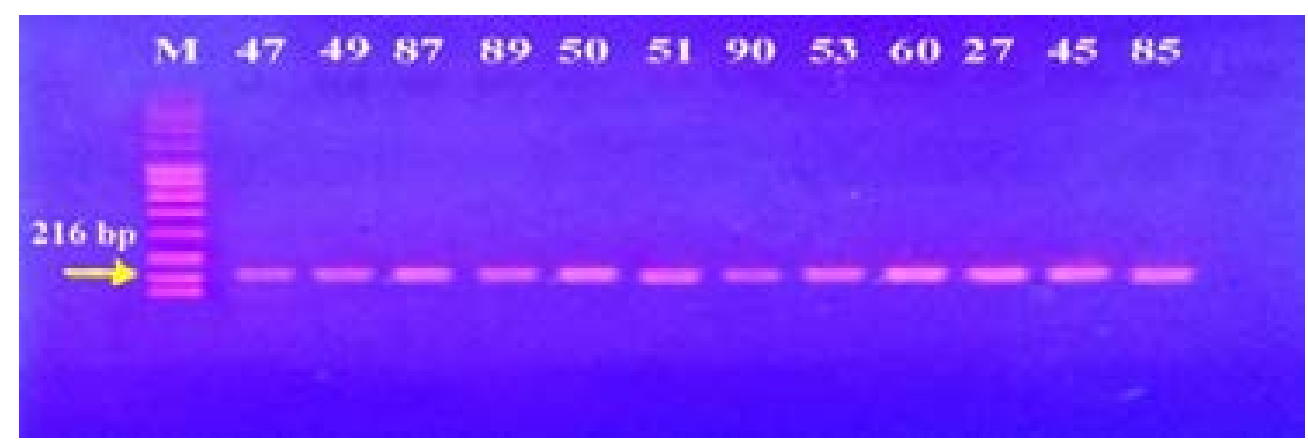

Figure 4. Electrophoresis of PCR products of norC gene. M; marker (100 bp). All isolates showed a band at 216 bp that corresponds to $\operatorname{nor} C$ gene. 


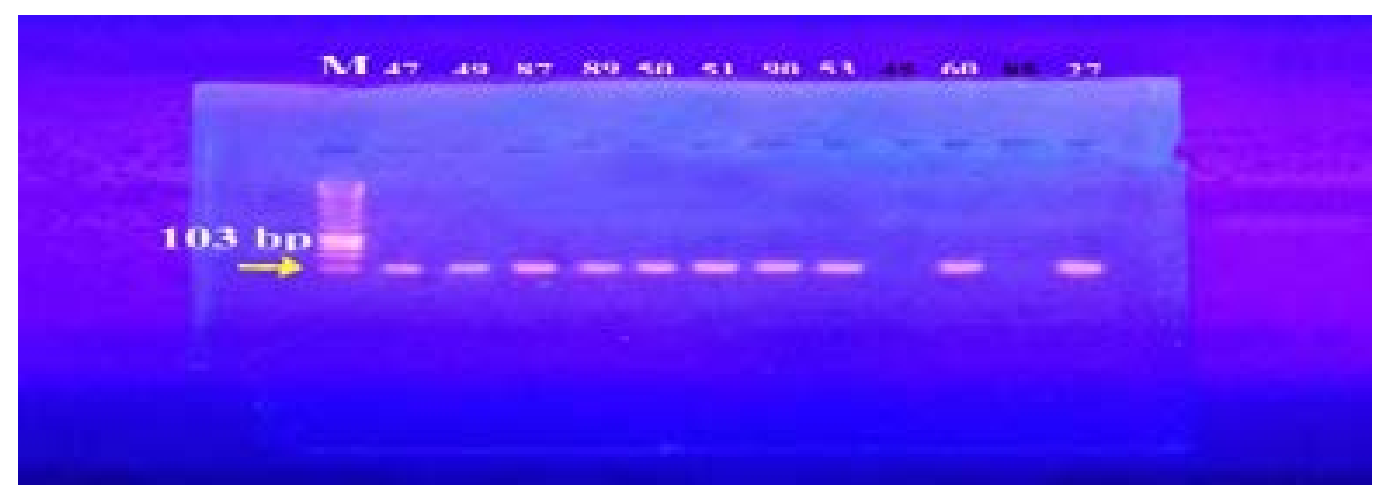

Figure 5. Electrophoresis of PCR products of sepA gene. M; marker (100 bp). Isolates $47,49,87,89,50,51,90,53,60$ and 27 showed a band at 103 bp that corresponds to sepA gene

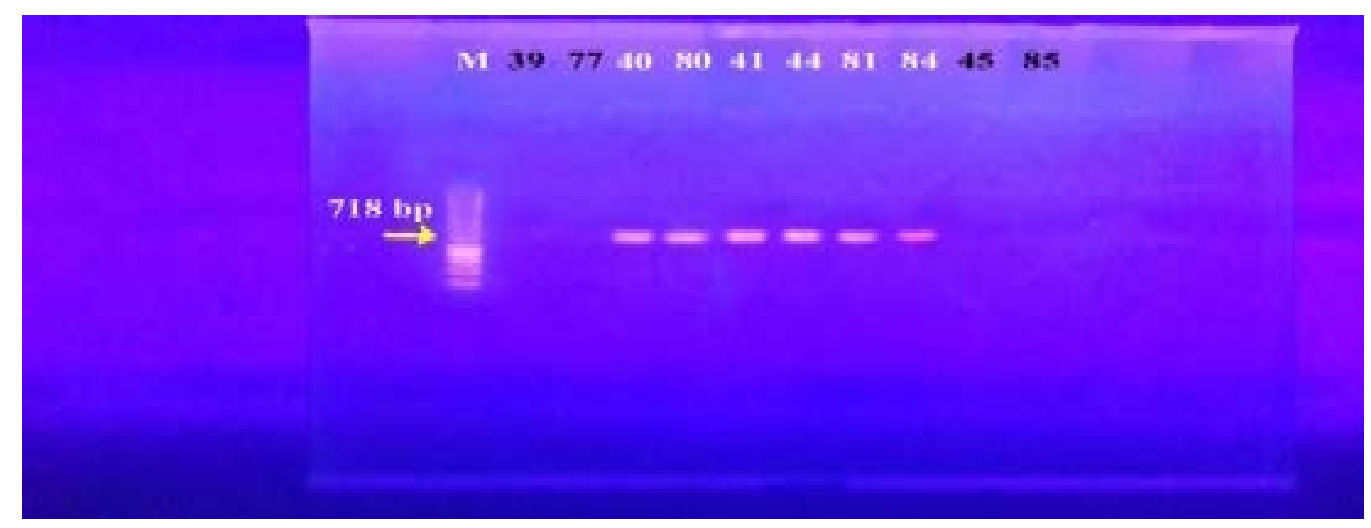

Figure 6. Electrophoresis of PCR products of mepA gene. M; marker (100 bp). Isolates $40,80,41,81,44$ and 84 showed a band at 718 bp that corresponds to mepA gene.

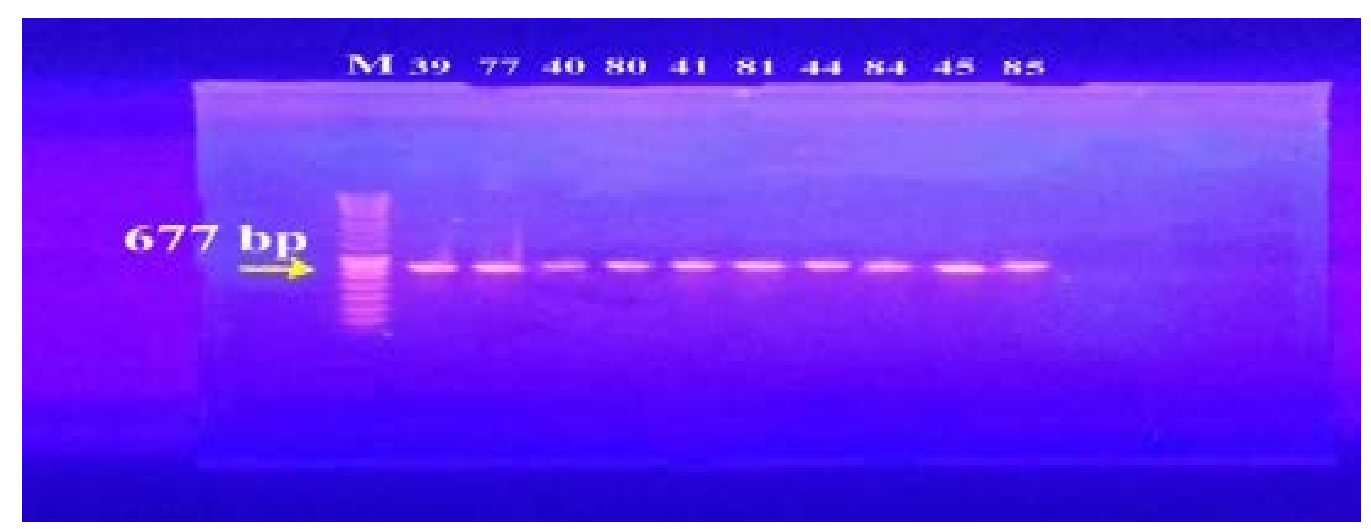

Figure 7. Electrophoresis of PCR products of $m d e A$ gene. M; marker (100 bp). All isolates showed a band at $677 \mathrm{bp}$ that corresponds to $m d e A$ gene. 


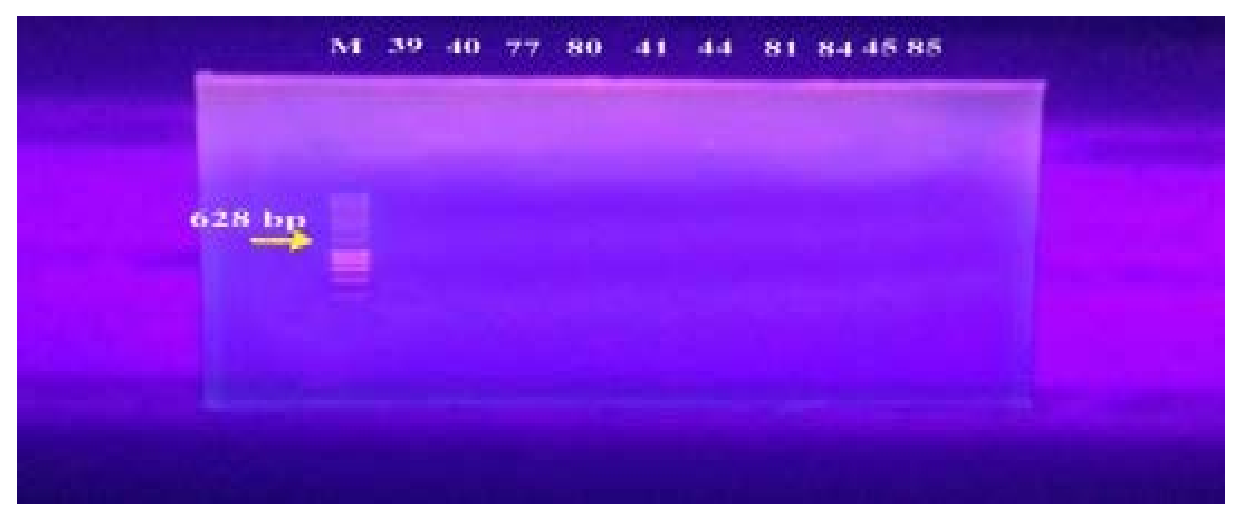

Figure 8. Electrophoresis of PCR products of $q a c A / B$ gene. M; marker (100 bp). All isolates showed no bands at $628 \mathrm{bp}$ that correspond to qacA/B gene.

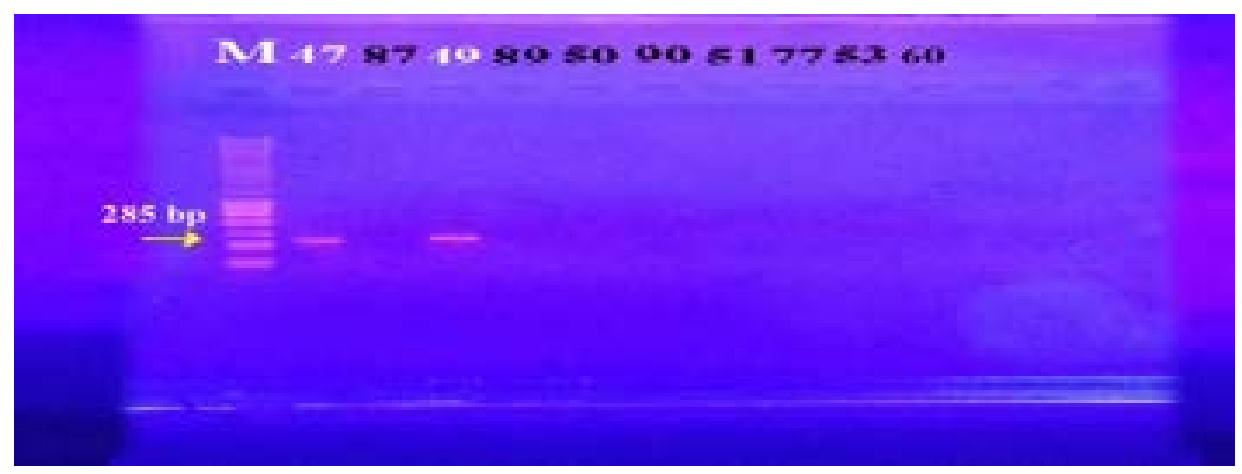

Figure 9. Electrophoresis of PCR products of $s m r$ gene. M; marker (100 bp). Isolates 47 and 49 showed a band at $285 \mathrm{bp}$ that corresponds to $s m r$ gene.

The most prevalent genes found in the isolates were the six chromosomal genes (mdeA, norB, norC, sepA, norA, mepA), that were found in $40-90 \%$ of the isolates, while the two plasmid genes (qacA/B and smr) were the least prevalent ones among the tested isolates (0-10\% of the isolates).

Assessment the effect of tested agents on the resistance of multi-drug resistant bacteria for efflux pumps by ethidium bromide agar cartwheel method
The cartwheel assay was used in the presence and absence of sub-MIC of selected drugs and the fluorescence was compared. Verapamil as a well reported efflux inhibitor increased the fluorescence indicating its effect on inhibition of efflux pumps, while mebeverine, vildagliptine, domperidone, nifuroxazide and atorvastatin showed no effect. Metformin was tested as efflux pump inhibitor in $2 \mathrm{MDR}$ isolates and it enhanced the fluorescence indicating a possible EPI activity (Fig. 10). 

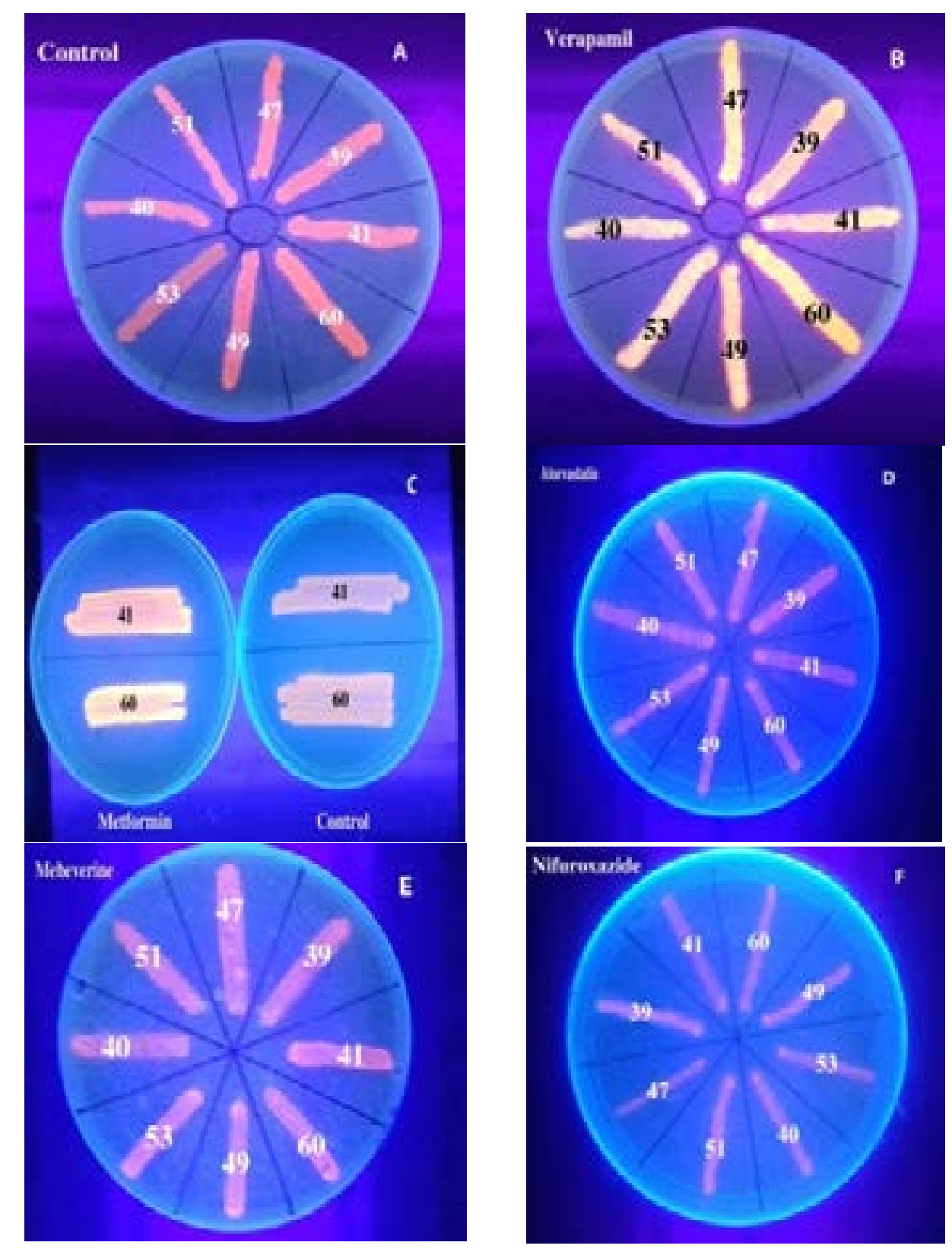

Figure 10. Effect of the tested agents on efflux pump by EtBrCW method A) Control plate with no drugs, B) Verapamil and C) Metformin enhanced the fluorescence indicating a possible EPI activity, D) Atorvastatin, E) Mebeverine and F) Nifuroxazide had no effect on fluorescence indicating no effect on efflux pump activity.

Effect of potential EPIs on the resistance of $S$. aureus isolates to different antimicrobial agents.

In order to confirm the results of $\mathrm{EtBrCw}$ method, the MICs of tested antibiotics and biocides were determined by broth microdilution method against $S$. aureus isolates in the presence of $1 / 4$ MIC (sub-MIC) of the tested agents that were positive for $\mathrm{EtBrCw}$ method (verapamil and metformin). The minimum inhibitory concentrations of the tested drugs were $0.25 \mathrm{mg} / \mathrm{ml}$ for nfuroxazide, $1 \mathrm{mg} / \mathrm{ml}$ for verapamil, mebeverine and atorvastatin, $8 \mathrm{mg} / \mathrm{ml}$ for vildagliptin and $32 \mathrm{mg} / \mathrm{ml}$ for each of metformin and domperidone. Sub-MICs of verapamil and metformin significantly reduced the MICs of antibiotics and biocides. Metformin showed much higher activity in reducing MICs (64-2048 fold decrease) as compared with the well-known efflux pump inhibitor verapamil (2-512 fold decrease) as shown in tables (3-6). 
Table 3. Synergistic activity of verapamil with antimicrobial agents against MDR S. aureus isolates

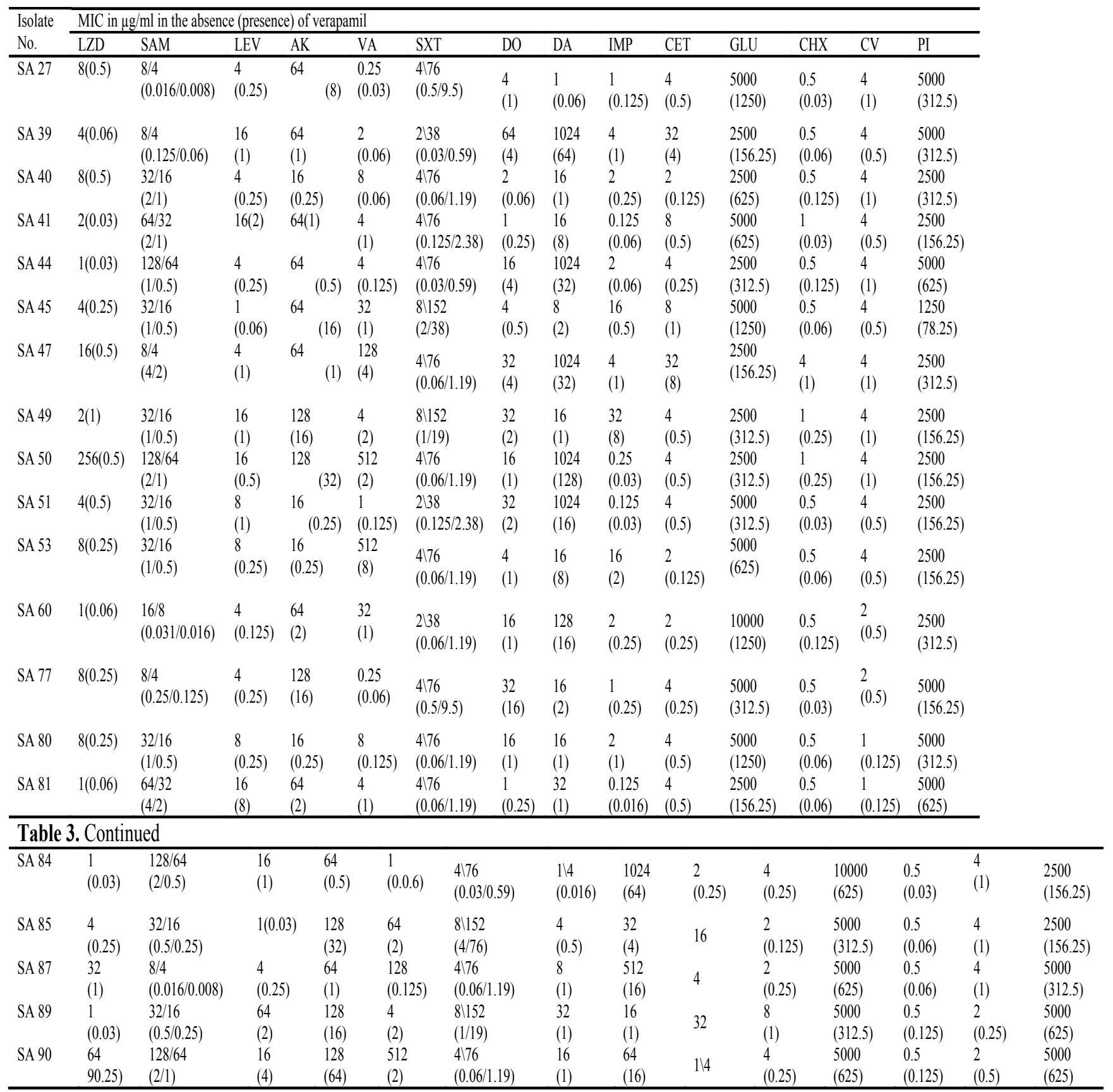

SA: Staphylococcus aureus, LZD: Linzeolid, SAM: Ampicillin-sulbactam, LEV: Levofloxacin, AK: Amikacin, VA: Vancomycin, SXT: Sulfamethoxazole-trimethoprim, DO: Doxycycline, DA: Clindamycin, IPM: Imipenem, CET: Cetrimide; GLU: Glutaraldehyde, CHX: Chlorhexidine; CV: Crystal violet, PI: Povidone-iodine. 
Table 4. Effect of verapamil on susceptibility of $S$. aureus to antimicrobial agents

\begin{tabular}{|c|c|c|c|c|c|c|c|c|c|c|c|c|c|c|}
\hline \multirow{2}{*}{$\begin{array}{l}\text { Isolate } \\
\text { No. }\end{array}$} & \multicolumn{14}{|c|}{ Folds decrease in MIC } \\
\hline & LZD & SAM & LEV & $\overline{\mathrm{AK}}$ & VA & SXT & $\mathrm{DO}$ & $\overline{\mathrm{DA}}$ & IMP & CET & $\overline{G L U}$ & $\mathrm{CHX}$ & $\mathrm{CV}$ & PI \\
\hline SA 27 & 16 & 512 & 16 & 8 & 8 & 8 & 4 & 16 & 8 & 8 & 4 & 16 & 4 & 16 \\
\hline SA 39 & 64 & 64 & 16 & 64 & 32 & 64 & 16 & 16 & 4 & 8 & 16 & 8 & 8 & 16 \\
\hline SA 40 & 16 & 16 & 16 & 64 & 128 & 64 & 32 & 16 & 8 & 16 & 4 & 4 & 4 & 8 \\
\hline SA 41 & 64 & 32 & 8 & 64 & 4 & 32 & 4 & 2 & 2 & 16 & 8 & 16 & 8 & 16 \\
\hline SA 44 & 32 & 128 & 16 & 128 & 32 & 128 & 4 & 32 & 32 & 16 & 8 & 4 & 4 & 8 \\
\hline SA 45 & 16 & 32 & 16 & 4 & 32 & 4 & 8 & 4 & 32 & 8 & 4 & 8 & 8 & 16 \\
\hline SA 47 & 32 & 2 & 4 & 64 & 32 & 64 & 8 & 32 & 4 & 4 & 8 & 4 & 4 & 8 \\
\hline SA 49 & 2 & 32 & 16 & 8 & 2 & 8 & 16 & 16 & 4 & 8 & 16 & 4 & 4 & 16 \\
\hline SA 50 & 512 & 64 & 32 & 4 & 256 & 64 & 16 & 8 & 8 & 8 & 8 & 4 & 4 & 16 \\
\hline SA 51 & 8 & 32 & 8 & 64 & 8 & 16 & 16 & 64 & 4 & 8 & 8 & 16 & 8 & 16 \\
\hline SA 53 & 32 & 32 & 32 & 64 & 64 & 64 & 4 & 2 & 32 & 16 & 16 & 8 & 8 & 16 \\
\hline SA 60 & 32 & 512 & 32 & 32 & 32 & 32 & 16 & 8 & 8 & 8 & 8 & 4 & 4 & 8 \\
\hline SA 77 & 16 & 32 & 16 & 8 & 4 & 8 & 2 & 8 & 4 & 16 & 8 & 16 & 4 & 32 \\
\hline SA 80 & 16 & 32 & 32 & 64 & 128 & 64 & 16 & 16 & 2 & 8 & 16 & 8 & 8 & 16 \\
\hline SA 81 & 32 & 16 & 2 & 32 & 4 & 64 & 4 & 32 & 8 & 8 & 4 & 8 & 8 & 8 \\
\hline SA 84 & 32 & 64 & 16 & 128 & 16 & 128 & 16 & 16 & 8 & 16 & 16 & 16 & 4 & 16 \\
\hline SA 85 & 16 & 64 & 32 & 4 & 32 & 2 & 8 & 8 & 16 & 16 & 16 & 8 & 4 & 16 \\
\hline SA 87 & 32 & 512 & 16 & 64 & 8 & 64 & 8 & 32 & 4 & 8 & 8 & 8 & 4 & 16 \\
\hline SA 89 & 32 & 64 & 32 & 8 & 2 & 8 & 32 & 16 & 4 & 8 & 16 & 4 & 8 & 8 \\
\hline SA 90 & 16 & 64 & 4 & 2 & 256 & 64 & 16 & 4 & 16 & 16 & 8 & 4 & 4 & 8 \\
\hline
\end{tabular}

SA: Staphylococcus aureus, LZD: Linzeolid, SAM: Ampicillin-sulbactam, LEV: Levofloxacin, AK: Amikacin, VA: Vancomycin, SXT: Sulfamethoxazole-trimethoprim, DO: Doxycycline, DA: Clindamycin, IPM: Imipenem, CET: Cetrimide; GLU: Glutaraldehyde, CHX: Chlorhexidine; CV: Crystal violet, PI: Povidone-iodine.

Table 5. Synergistic activity of metformin with antimicrobial agents against representative MDR S. aureus isolates

\begin{tabular}{lllllllllllllll}
\hline Isolate & \multicolumn{1}{l}{ MIC in $\mu \mathrm{g} / \mathrm{ml}$ in the absence (presence) of verapamil } \\
\cline { 2 - 7 } No. & LZD & SAM & LEV & AK & VA & SXT & D0 & DA & IMP & CET & GLU & CHX & CV & PI \\
\hline SA 41 & 2 & $64 / 32$ & 16 & 64 & 4 & 4176 & 1 & 16 & 0.125 & 8 & 5000 & 1 & 4 & 2500 \\
& $(0.002)$ & $(0.06 / 0.03)$ & $(0.016)$ & $(0.03)$ & $(0.004)$ & $(0.004 / 0.074)$ & $(0.001)$ & $(0.008)$ & $(0.0001)$ & $(0.06)$ & $(78.125)$ & $(0.002)$ & $(0.06)$ & $(19.53)$ \\
SA 60 & 1 & $16 / 8$ & 4 & 64 & 32 & 2138 & 16 & 128 & 2 & 2 & 10000 & 0.5 & 2 & 2500 \\
& $(0.001)$ & $(0.016 / 0.008)$ & $(0.002)$ & $(0.06)$ & $(0.016)$ & $(0.008 / 0.037)$ & $(0.016)$ & $(0.125)$ & $(0.002)$ & $(0.016)$ & $(39.06)$ & $(0.008)$ & $(0.08)$ & $(39.06)$ \\
\end{tabular}

SA: Staphylococcus aureus, LZD: Linzeolid, SAM: Ampicillin-sulbactam, LEV: Levofloxacin, AK: Amikacin, VA: Vancomycin, SXT: Sulfamethoxazole-trimethoprim, DO: Doxycycline, DA: Clindamycin, IPM: Imipenem, CET: Cetrimide; GLU: Glutaraldehyde, CHX: Chlorhexidine; CV: Crystal violet, PI: Povidone-iodine.

Table 6. Effect of metformin on susceptibility of S. aureus to antimicrobial agents

\begin{tabular}{|l|l|l|l|l|l|l|l|l|l|l|l|l|l|l|}
\hline IsolateNo. & \multicolumn{10}{|l|}{ Fold decrease in MIC } \\
\cline { 2 - 14 } & LZD & SAM & LEV & AK & VA & SXT & D0 & DA & IMP & CV & CET & GLU & CHX & PI \\
& & & & & & & & & & & & & & \\
\hline SA 41 & 1024 & 1024 & 1024 & 2048 & 1024 & 1024 & 1024 & 2048 & 1024 & 64 & 128 & 64 & 512 & 128 \\
\hline SA 60 & 2048 & 1024 & 2048 & 1024 & 2048 & 1024 & 1024 & 1024 & 1024 & 256 & 128 & 256 & 64 & 64 \\
\hline
\end{tabular}

SA: Staphylococcus aureus, LZD: Linzeolid, SAM: Ampicillin-sulbactam, LEV: Levofloxacin, AK: Amikacin, VA: Vancomycin, SXT: Sulfamethoxazole-trimethoprim, DO: Doxycycline, DA: Clindamycin, IPM: Imipenem, CET: Cetrimide; GLU: Glutaraldehyde, CHX: Chlorhexidine; CV: Crystal violet, PI: Povidone-iodine.

\section{Discussion}

The global crisis of the emergence of antibiotic resistance among bacteria, especially multidrug resistance
(MDR) made it urgent to discover new antibacterials and agents that can play as resistance modifiers (EPIs). Efflux pumps have the ability to expel structurally unre- 
lated compounds, including antibiotics used in a clinical setting and hence antibiotics lost their clinical efficacy. The over-expression of efflux pumps can lead to rapid development of antibiotic resistance. This can take place even to the antibiotics of last resort. It is therefore necessary to investigate the ability of new drugs to act as efflux pump inhibitors (EPIs). By the introduction of EPIs, the traditional therapeuticaly ineffective antibiotics could find its way back to clinical use ${ }^{22,23}$.

Efflux systems in Staph. aureus are critical for exporting antibiotics and detergents ${ }^{4}$. Therefore, the selected MDR isolates in this study were screened phenotypically for efflux pumps by $\mathrm{EtBrCw}$ method and genotypically by PCR.

Active efflux was detected in all isolates by the cartwheel assay. This was in accordance with Rana et al. ${ }^{24}$ in which all MDR Staphylococcal isolates showed active efflux.

In order to confirm phenotypic study of efflux pump as a resistance mechanism, genotypic investigation of efflux pump genes was done. PCR amplification technique was performed to detect the prevalence of norA, norB, norC, sepA, mepA, mdeA, qacA/B and smr geneslux mediated resistance ${ }^{8}$.

The study revealed that mdeA and norB genes were the most frequent ones (90\%), while qacA/B gene was absent in all isolates. This was more or less similar to the results reported in Iran by Sepideh et al. ${ }^{13}$, in which the most prevalent gene was mdeA (61.7\%), while norB gene was present in $41.7 \%$ of isolates. Moreover, qacA/B gene was present in only $3.3 \%$ of isolates. Furthermore, a canadian report stated that the distribution of qacA/B was only $2 \%{ }^{25}$. In contrast, our results were much lower than an Asian study that reported prevalence rates of $73 \%$ for the qacA/B26. Moreover, the prevalence rates of norA, norC, sepA and mepA genes were $75 \%, 80 \%, 80 \%$ and $40 \%$, respectively. However, the study of Sepideh et al.13 reported lower prevalence rates; $41.7 \%$, for both of norA and norC genes and $35 \%$ for sepA, while the rate of presence of mepA was higher than this study $(60 \%)$.

Smr gene was found in 10\% of isolates only. These results were in agreement with that obtained by Longtin et al. ${ }^{25}$ in Canada that reported prevalence rate of $7 \%$. In contrast, these results were lower than a study in Asia that reported prevalence rate of $32 \%$ for smr gene ${ }^{26}$.

From the obtained results it might be concluded that genes mdeA, norB, norC, norA and sepA play the major role in efflux, while genes mepA, smr and qacA/B play a minor role in efflux. Strong correlation between active efflux as a resistance mechanism and antimicro- bials MDR found in this study was supported by Costa et al. ${ }^{14}$,who reported that active efflux is a major mechanism in the early stages of resistance development. The variation in the frequency of efflux genes among different studies may be due to temporal and geographical variation ${ }^{27}$.

To combat efflux-mediated resistance, natural or synthetic compounds were investigated in previous studies and many compounds were proved to have inhibitory activities against efflux pumps in $S$. aureus. The TetK efflux-pump in $S$. aureus was blocked by the essential oil of Chenopodium ambrosioides $L$. and its $\alpha$-terpinene active ingredient ${ }^{28}$. Moreover, the natural compound Juglone could reverse the efflux-mediated resistance of $S$. aureus to tetracycline, erythromycin and benzalkonium chloride $^{29}$. Some synthetic amino acid amides of piperic acid and 4-ethylpiperic acid were reported to have efflux inhibiting activity in $\mathrm{S}$. aureus and when used in combination with ciprofloxacin, synergism was found $\mathrm{d}^{30}$.

In our study, some FDA approved drugs such as verapamil, metformin, mebeverine, domperidone, vildagliptin, nifuroxazide and atorvastatin were tested for their potential efflux pump inhibiting activities. Mebeverine, domperidone, vildagliptin, nifuroxazide and atorvastatin showed no effect on the fluorescence of $\mathrm{EtBr}$ indicating no EPI activity upon screening by the cartwheel method. On the other hand, verapamil and metformin enhanced the fluorescence indicating their effects on inhibition of efflux pumps. Verapamil as well reported EPI causes accumulation of EtBr within the cells and induce fluorescence at a much lower concentration of EtBr. Metformin was reported as an effective inhibitor of P-glycoprotein expression and MDR reversing agent ${ }^{31}$. Metformin can decrease the consumption of oxygen in mitochondria and alter the distribution of charge in addition to the membrane charge. Furthermore, it was found to interfere with the transport of protons and other cations across membranes. Metformin was found beneficial in preventing breast cancer cell growth through augmenting the cytotoxicity of chemotherapeutic agents in tumor cells and reducing the high levels of MDR1 mRNA and protein in these cells ${ }^{32}$.

In order to confirm the effect of the well reported EPI verapamil, and the suggested one metformin on efflux mechanism in the selected MDR isolates, the MICs of antibiotics and biocides were detected in the presence and absence of sub-inhibitory concentrations of the drugs. In general, the tested agents caused significant reduction in MICs with variable degrees. In order to screen the effect of EPIs within these ranges, fold decrease in MICs exhibited by $50 \%$ of isolates was calculated to compare the potency of EPIs. 
Metformin was more potent than verapamil. Metformin reduced MICs of all tested antibiotics by 1024-2048 folds. Additionally, it reduced MICs of all tested biocides by 64-512 folds. Moreover, verapamil reduced the MICs of $\beta$-lactams, vancomycin and linezolid by 32 folds. Moreover, it reduced the MICs of levofloxacin, clindamycin and doxycycline by 16 folds and MICs of amikacin and sulfamethoxazole-trimethoprim by 64 folds. Additionally, it reduced the MICs of povidone iodine, cetrimide, glutaraldehyde and chlorhexidine by 8 folds and crystal violet by 4 folds. These findings were supported by El-Domany ${ }^{33}$, who reported that combination of verapamil at sub MICs with $\beta$-lactams, fluoroquinolones and tetracyclines resulted in sharp reduction in the MICs ranged between 8 and 128 folds. Also, our findings were supported by $\mathrm{Gabr}^{34}$, who found that the MICs decrease with the use of verapamil ranged between 8-64-folds for $\beta$-lactams, 4-32 folds for ciprofloxacin, 8-32-folds for macrolides, gentamicin and tetracycline. Additionally, Gabr34 found that MICs decrease with the use of verapamil were 4-16 folds for povidone iodine, cetrimide and chlorhexidine in accordance with our results.

A study conducted by $\mathrm{Abbas}^{35}$ revealed that the MICs of tested agents (azithromycin, crystal violet, ciprofloxacin, cefoperazone, tetracycline and streptomycin) were reduced up to 64 folds in the presence of verapamil. This sharp reduction in MICs of antimicrobials upon combination with the efflux inhibitors suggested presence of efflux pumps that extrude these antimicrobials out of the cells of multi-drug resistant isolates. This may reveal that efflux activity has a strong involvement in the reduced susceptibility to antimicrobials ${ }^{13}$.

\section{Conclusion}

It could be concluded that active efflux plays a great role in multi-drug resistance as investigated phenotypically and genotypically. This study also highlighted the positive significant effect of EPIs to combat the high multi-drug resistance to antimicrobial.

Further work is needed to confirm the effect of efflux inhibitors on the expression of efflux pump genes by realtime PCR and to investigate their effects in vivo.

\section{Conflict of interest}

None declared.

\section{References}

1. Centers for Disease Control and Prevention. Office of Infectious Disease Antibiotic resistance threats in the United States, 2013.

2. Vranakis I, Goniotakis I, Psaroulaki A, Sanda- lakis V, Tselentis Y, Gevaert K, Tsiotis G. Proteome studies of bacterial antibiotic resistance mechanisms. Journal of Proteomics 2014; 97(5): 88-99.

3. Li XZ, Nikaido H. Efflux-mediated drug resistance in bacteria. Drugs 2009; 69(12): 1555-1623.

4. Ahmad B, Urbas F, Jamil J, Ahmed J, Bashir S. Biocides susceptibility pattern and phenotypic detection of Efflux pump in Staphylococcus aureus isolates from two tertiary hospitals of Pakistan. African Journal of Microbiology Research 2013; 7(25): 3171-3178.

5. Li XZ, Nikaido H. Efflux-mediated drug resistance in bacteria. Drugs 2004; 64(2): 159-204.

6. Handzlik J, Matys A, Keć-Kononowicz K. Recent advances in multi-drug resistance (MDR) efflux pump inhibitors of Gram-positive bacteria Staph. aureus. Antibiotics 2013; 2(1): 28-45.

7. Mikulášová M, Chovanová R, Vaverkova S. Synergism between antibiotics and plant extracts or essential oils with efflux pump inhibitory activity in coping with multidrug-resistant staphylococci. Phytochemistry Reviews 2016; 15: 651-662.

8. Diniz-Silva HT, Magnani M, Siqueira S, de Souza EL, de Siqueira-Junior JP. Fruit flavonoids as modulators of norfloxacin resistance in Staphylococcus aureus that overexpresses norA LWT-Food and Science Technology 2017; 85: 324-326.

9. Lima VN, Oliveira-Tintino CDM, Santos ES, Morais LP, Tintino SR, Freitas TS, Geraldo YS, Pereira RLS, Rafael P Cruz RP, Menezes IRA, Coutinho HDM. Antimicrobial and enhancement of the antibiotic activity by phenolic compounds: gallic acid, caffeic acid and pyrogallol. Microbial Pathogenesis 2016; 99: 56-61.

10. Tintino SR, Oliveira-Tintino CDM , Campina FF, Silva RLP, Costa MDS, Menezes IRA, Calixto-Júnior JT, Siqueira-Junior JP, Coutinho HDM, Leal-Balbino TC, Balbino VQ. Evaluation of the tannic acid inhibitory effect against the NorA efflux pump of Staphylococcus aureus. Microbial Pathogenesis 2016; 97 : 9-13.

11. Pereira PS, deLima MDCA, Neto PPM, Oliveira-Tintino CDM, Tintino SR, Menezes IRDA, Jamerson Ferreirade Oliveira JF, Marchand P, Coutinho HDM, Rodrigues MDD, Silva TG. Thiazolidinedione and thiazole derivatives potentiate norfloxacin activity against NorA efflux pump over expression in Staphylococcus aureus 1199B strains. Bioorganic Medicical Chemistry 2019; 27(17):3797-380.

12. Abbas HA, Shaker GH, Hegazy WA, Baiomy AA. Prevalence of multi-drug resistant Staphylococci isolated from surgical site infections. Zagazig Journal of Pharmacentical Sciences 2018; 27(1): 31-38.

13. Sepideh H, Rahil M, Masoud Y, Emran A, 
Askari E , Saniei M , M Pourmand MR. Frequency of efflux pump genes mediating ciprofloxacin and antiseptic resistance in methicillin-resistant Staphylococcus aureus isolates. Microbial Pathogenesis 2017; 111: 71-74.

14. Costa S, Junqueira E, Palma C, Viveiros M, Melo-Cristino J, Amaral L, Couto I. Resistance to antimicrobials mediated by efflux pumps in Staphylococcus aureus. Antibiotics 2013; 2(1): 83-99.

15. Martins M, McCusker MP, Viveiros M, Couto I, Fanning S, Pagès J, Amaral L. A simple method for assessment of MDR bacteria for over-expressed efflux pumps. The Open Microbiology Journal 2013; 7: 72-83.

16. Bjorland SM, Waage S. Plasmid-borne smr gene causes resistance to quaternary ammonium compounds in bovine Staphylococcus aureus. Journal of Clinical Microbiology 2001; 39(11): 3999-4004.

17. Couto I, Costa SS, Viveiros M, Martins M, Amaral L. Efflux-mediated response of Staphylococcus aureus exposed to ethidium bromide. Journal of Antimicrobial Chemotherapy 2008; 62(3): 504-513.

18. Nair MK, Venkitanarayanan KS. Cloning and sequencing of ompA gene of Enterobacter sakazakkii and development of an ompA-targeted PCR for rapid detection of Enterobacter sakazakii in infant formula. Applied and Environmental Microbiology 2006; 72: 25392546.

19. Sambrook J, Russell DW. Molecular Cloning: A Laboratory Manual. 3rd Ed. Cold Spring Harbor Laboratory, Cold Spring Harbor Press, New York, USA. 2001; 262-272.

20. CLSI-Clinical and Laboratory Standards Institute. Methods for dilution antimicrobial susceptibility tests for bacteria that grow aerobically: Approvated standard, CLSI Document M07-A9. Wayne, USA, 2012. 21. Vicki AL, Tony JH, Debbie SK, Andrew CC. Susceptibility of 169 USA300 methicillin-resistant Staphylococcus aureus isolates to two copper-based biocides, CuAL42 and CuWB50. Journal of Antimicrobial Chemotherapy 2010; 65: 939-941.

22. Stavri M, Piddock LJ, Gibbons S. Bacterial efflux pump inhibitors from natural sources. Journal of Antimicrobial Chemotherapy 2007; 59, 1247-1260.

23. Piddock LJV. Multidrug-resistance efflux pumps not just for resistance Nature Reviews of Microbiology 2006; 4:629-636.

24. Rana T, Kaur N, Farooq U, Khan A, Singh S. Efflux as an arising cause of drug resistance in Punjab (India). IJBPAS 2015; 4(9):

25. Longtin J, Christine S, Krystal S, Allison M, Simor A, Longtin Y, Low DE, Melano RG. Distribution of antiseptic resistance genes QacA, QacB, and Smr in methicillin resistant Staphylococcus aureus isolated in Toronto, Canada, from 2005 to 2009. Antimicrobial Agents Chemotherapy 2011; 55(6): 2999-3001.

26. Sheng WH, Wang JT, Lauderdale TL. Epidemiology and susceptibilities of methicillin-resistant Staphylococcus aureus in Taiwan: emphasis on chlorhexidine susceptibility. Diagnostic Microbiology and Infectious Disease 2009; 63(3): 309-313. 5967-5979.

27. Kosmidis C, Schindler BD, Jacinto PL, Patel D, Bains K, Susan M, Seo SM, Kaatz GW. Expression of multidrug resistance efflux pump genes in clinical and environmental isolates of Staphylococcus aureus. International Journal of Antimicrobial Agents 2012; 40 (3): 204209.

28. Limaverde PW, Campina FF, da Cunha FAB, Crispim FD, Figueredo FG, Lima LF, Oliveira-Tintino CDM, de Matos YMLS, Morais-Braga MFB, Menezes IRA, BalbinoMahdouani K, Chaieb K. Use of juglone as antibacterial and potential efflux pump inhibitors in Staphylococcus aureus isolated from the oral cavity. Microbial Pathogenesis 2016;101:44-49.

29. Wani NA, Singh S, Farooq S, Shankar S, Koul $S$, Khan IA , Rai R. Amino acid amides of piperic acid (PA) and 4-ethylpiperic acid (EPA) as NorA efflux pump inhibitors of Staphylococcus aureus. Bioorganice Medicinal Chemistry Letters. 2016; 26(17): 4174-4178.

30. Kim HG, Hien TT, EH, Hwang YP, Choi JH, K Kang KW, Kwon K, Kim B, Kim SK, G Song GY, Jeong TC, Jeong HG. Metformin inhibits P-glycoprotein expressionvia the NF-kB pathway and CRE transcriptional activity through AMPK activation. British Journal of Pharmacology 2011; 162:1096-1108.

31. Vazquez-Martin A, Oliveras-Ferraros C, del Barco S, Martin-Castillo B, Menendez JA. The antidiabetic drug metformin: a pharmaceutical AMPK activator to overcome breast cancer resistance to HER2 inhibitors while decreasing risk of cardiomyopathy. $A n$ nals of Oncology 2009; 20(3): 592-595.

32. El-Domany RA. Effect of different efflux inhibitors on $\beta$-lactams resistance in multi-drug resistant Staphylococcus aureus. Egyptian Journal of Medical Microbiology 2007; 16(3): 429-436.

33. Gabr IM. (2016). Studies on multi-drug resistant bacteria isolated from burn wound infections. MSC thesis, Department of Microbiology and Immunology, Zagazig University, Zagazig, Egypt, 2016.

34. Abbas HA. Inhibition of hemolysin transporter protein by multi-drug efflux inhibitors in Escherichia Coli. MSC thesis, Department of Microbiology and Immunology, Zagazig University, Zagazig, Egypt, 2006. 